Z Herz- Thorax- Gefäßchir 2022 · 36:97-99 https://doi.org/10.1007/s00398-021-00483-6 Angenommen: 9. Dezember 2021 Online publiziert: 1. Februar 2022

(C) The Author(s), under exclusive licence to Springer Medizin Verlag $\mathrm{GmbH}$, ein Teil von Springer Nature 2022

\section{Handlungsalgorithmus: ECMO/ECLS bei SARS-CoV-2 Disease}

\author{
Udo Boeken · Payam Akhyari' · Andreas Beckmann \\ ${ }^{1}$ Klinik für Herzchirurgie, Universitätsklinikum Düsseldorf, Düsseldorf, Deutschland
}

Seit Ausbruch der weltweiten SARS-CoV2-Pandemie hat der Einsatz extrakorporaler Lungen-/Herzunterstützungssysteme bei pulmonal bzw. kardiopulmonal akut insuffizienten Patienten quantitativ erheblich zugenommen. Hierfür sind, im Kontext der generellen Verfügbarkeit von Intensivstationskapazitäten, noch limitiertere Ressourcen vorhanden. Ferner gilt es zu berücksichtigen, dass nach gegenwärtigen wissenschaftlichen Erkenntnissen, beispielsweise aus internationalen Multizenterregistern, der Erfolg der ECMO-/ECLS-Therapie bei SARS-CoV2-assoziierter akuter Lungen- und Herzinsuffizienz ernüchternd ist [1]. Vor diesem Hintergrund zielt dieser Beitrag darauf $a b$, auf Basis aktueller Kenntnisse einen Entscheidungs- und Handlungsalgorithmus (- Abb. 1) für die Praxis zu entwerfen.

1. Patientencharakteristika: Erwachsene(r) mit nachgewiesener COVID-19Erkrankung und klinisch manifester (kardio-)pulmonaler Insuffizienz (Hypoxie oder/und Hyperkapnie; Kreislaufinstabilität)

2. Vor Indikationsstellung:

- Erfassung relevanter Komorbiditäten,

- Erfassung relevanter Laborparameter $^{1}$

- Behandlung ggf. vorliegender Grunderkrankungen,

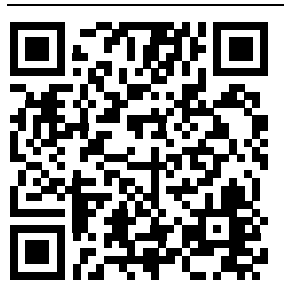

QR-Code scannen \& Beitrag online lesen
- lungenprotektive Beatmung/ Optimierung des Kreislaufs,

- Eruierung relativer/absoluter Kontraindikationen ${ }^{2}$.

3. Pulmonale Situation

1. Horovitz-Index $<150 \mathrm{~mm} \mathrm{Hg}$ : primäre Oxygenierungsstörung

- $\quad$ Optimierung der Beatmung durch

a. Lagerung: Bauchlage mindestens $16 \mathrm{~h}$

b. Ventilation: DUOPAP, HighPEEP (Wert?), Rekrutierungsmanöver, ggf. NO,

c. Medikation: suffiziente Relaxierung, adäquate Analogosedierung,

2 Kontraindikationen der ECMO-/ECLS-Therapie bei SARS-CoV-2-assoziiertem Lungenversagen:

relative Kl: Alter $>65 \mathrm{~J}$., $\mathrm{BMI} \geq 40$, unklarer Patientenwille, fortgeschrittene Herzinsuffizienz, hochdosierte Vasopressortherapie (ohne vaoder vva-Therapieoption), reduzierter Immunstatus;

absolute KI: hohes Alter, reduzierter AZ (Clinical Frailty Scale Category $\geq 3$ ), invasive Beatmung $>7 \mathrm{~T}$, relevante Komorbiditäten (CKD $\geq$ III, Child-Pugh-Klasse B/C, Demenz, fortgeschrittene maligne Erkrankung, fortgeschrittene Lungenerkrankung, schlecht eingestellter Diabetes mellitus $\left(\mathrm{HbA}_{1 \mathrm{c}}>9 \%\right)$ mit Endorganschäden, Mangelernährung (Body-Mass-Index $<18,5 \mathrm{~kg} / \mathrm{m}^{2}$ ), schwere pAVK, limitierte Lebenserwartung [präexistente Pflegebedürftigkeit], fortgeschrittenes Multiorganversagen, schwerer neurologischer Schaden, nicht ursächlich therapierbare Blutung, Kontraindikation für Antikoagulation, repetitiver Bluttransfusionsbedarf, fortwährende CPR). 


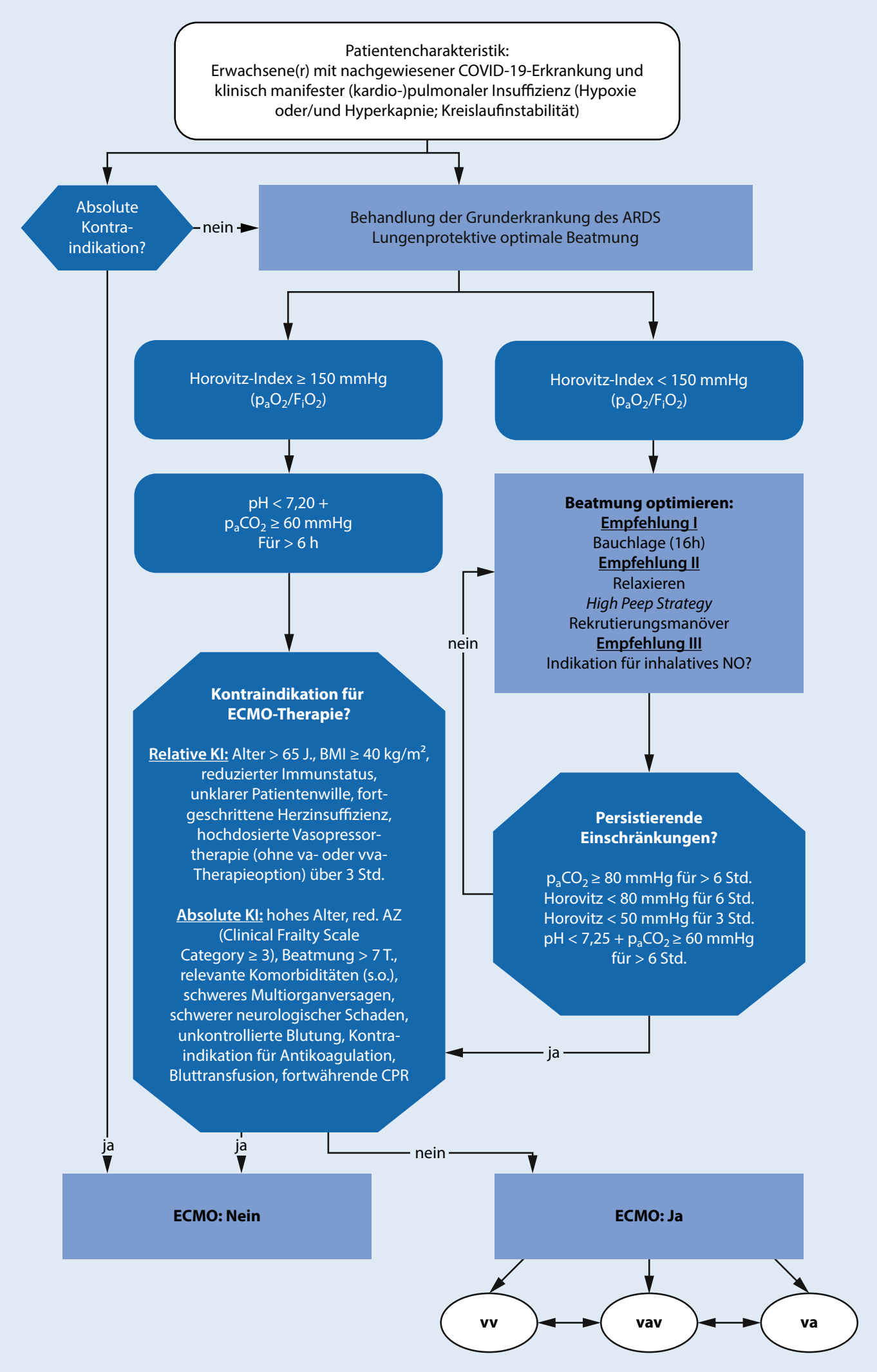

Abb. $1 \triangleleft$ Handlungsalgorithmus ECMO-Therapie bei COVID-19-assoziiertem Lungenversagen. ECMO extrakorporale Membranoxygenierung, $v v$ venovenös, vav venoarteriell-venös, va venoarteriell, $B M I$ body mass index, KI Kontraindikation, CPR cardiopulmonale Reanimation, NOStockstoffmonoxid 
- Zeitintervalle arterielle BGA: mindestens $1 / \mathrm{h}$ besser $1 / 30 \mathrm{~min}$,

- bei Progression der Oxygenierungsstörung (Horovitz $<80 \mathrm{mmHg}$ für $>6 \mathrm{~h}$ oder $<50 \mathrm{~mm} \mathrm{Hg}$ für $>3 \mathrm{~h}$ ),

- Evaluation von Kontraindikationen für ECMO/ECLS (absolut/relativ, s. unten), Initiierung einer ECMO-/ECLSImplantation (peripher/zentra nach klinischem Bild sowie patientenindividueller Situation).

2. Horovitz-Index $>150 \mathrm{~mm} \mathrm{Hg}$ : primäre Decarboxylierungsstörung, Hyperkapnie: $p H$ $<7,2$ und $p_{a} \mathrm{CO}_{2}[\mathrm{mmHg}] \geq 60$ (80, s. unten) für $>6 \mathrm{~h}$,

- $\quad$ Evaluation von Kontraindikationen für ECMO/ECLS (absolut/relativ, s. unten), Initiierung einer ECMO-/ECLSImplantation (peripher/zentral nach klinischem Bild sowie patientenindividueller Situation).

4. Gleichzeitige Herz-Kreislauf-Insuffizienz mit deutlicher Inotropikapflichtigkeit

- Evaluation von Kontraindikationen für ECLS (absolut/relativ, s. unten), insbesondere auch bezüglich peripherer/zentraler arterieller Kanülierung (pAVK, höhergradige Aortenklappeninsuffizienz),

- Implantation ECLS wie ECMO, jedoch distale Perfusionskanüle bei peripherer Kanülierung (Cave: HarlekinPhänomen),

- bei fortbestehender Hypoxie ( $3 \mathrm{~h}$ bei $<50 \mathrm{~mm} \mathrm{Hg}$ oder $6 \mathrm{~h}$ bei $<80 \mathrm{~mm} \mathrm{Hg}$ ) Prüfung einer Ausweitung auf vvaECLS.

\section{Korrespondenzadresse}

\section{Prof. Dr. Payam Akhyari}

Klinik für Herzchirurgie, Universitätsklinikum Düsseldorf

Düsseldorf, Deutschland

payam.akhyari@med.uni-duesseldorf.de

\section{Einhaltung ethischer Richtlinien}

Interessenkonflikt. U. Boeken, P. Akhyari und A. Beckmann geben an, dass kein Interessenkonflikt besteht.

Für diesen Beitrag wurden von den Autoren keine Studien an Menschen oder Tieren durchgeführt. Für die aufgeführten Studien gelten die jeweils dort angegebenen ethischen Richtlinien.

\section{Literatur}

1. Shekar K, Badulak J, Peek G, Boeken U, Dalton HJ, Arora L, Zakhary B, Ramanathan K, Starr J, Akkanti B, Antonini MV, Ogino MT, Raman L, Barret N, Brodie D, Combes A, Lorusso R, MacLaren G, Müller T, Paden M, Pellegrino V, ELSO Guideline Working Group. Extracorporeal Life Support Organization Coronavirus Disease (2020) 2019 Interim Guidelines: A Consensus Document from an International Group of Interdisciplinary Extracorporeal Membrane Oxygenation Providers. ASAIO J 66(7):707-721 (Jul)

\section{Autoren WERKSTATT}

GRATIS

Ein Service für Autoren von Springer Medizin

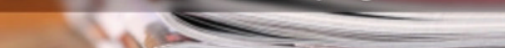

\section{Fortbildungen für Autor*innen und Gutach- ter*innen}

Die ersten Veröffentlichungen sind für Jeden ein wichtiger Schritt in der angestrebten Karriere. Wissenschaftliche Artikel sind entscheidend dafür, dass die eigene Arbeit in der Community wahrgenommen wird. Es geht darum, die eigenen Ideen national und international auszutauschen und sicherzustellen, dass die Ergebnisse Wirkung erzielen.

Die Online-Kurse der Autorenwerkstatt helfen, sich leicht einen Überblick über das Schreiben, Einreichen, Begutachten und Veröffentlichen eines Manuskripts zu verschaffen.

5 Online-Kurse zu den wichtigsten Standards des wissenschaftlichen Publizierens:

- Wie verfasse ich ein Manuskript?

- Writing in English für deutschsprachige Autorinnen und Autoren

- Wie funktionieren Publikation und Begutachtung?

- Anleitung zur Open-AccessVeröffentlichung

- Leitfaden zur Peer-ReviewBegutachtung

Für alle, die auf SpringerMedizin.de registriert sind!

Jetzt gratis fortbilden unter www.springermedizin.de/ autorenwerkstatt/ 